\title{
Ventricular Ablation
}

National Cancer Institute

\section{Source}

National Cancer Institute. Ventricular Ablation. NCI Thesaurus. Code C80433.

A invasive therapeutic procedure to destroy tissue in the cardiac ventricles that is the source of an arrhythmia. 